\title{
Quantenstatistische Behandlung des inneren Photoeffekts
}

\author{
Von Helmut Müser
}

\author{
Aus dem Physikalischen Institut der Universität Frankfurt a. Main \\ (Z. Naturforschg. 7 a, 729-734 [1952]; eingegangen am 16. Juli I952)
}

\begin{abstract}
Die Fermi-Verteilung gibt die Verteilung von Elektronen über die verschiedenen Energieniveaus in einem Festkörper unter der Voraussetzung an, daß sich ein thermodynamischer Gleichgewichtszustand eingestellt hat. Bei der Untersuchung von Leitfähigkeitsproblemen werden geringfügige Störungen dieser Gleichgewichtsverteilung durch elektrische Felder, Temperaturgefälle u. dgl. berücksichtigt. Der innere Photoeffekt bewirkt eine kräftige Störung der Gleichgewichtsverteilung, da fortgesetzt lichtelektrisch ausgelöste Elektronen aus einem niederen in einen höheren Energiezustand gehoben werden.

Im folgenden wird ein statistischer Ansatz für die Verteilung von Elektronen über dargebotene Energiestufen versucht für den Fall, daß laufend je Sekunde eine bestimmte Anzahl aus einem tieferen in ein höheres Niveau gehoben wird.
\end{abstract}

\section{Der allgemeine Ansatz}

$\mathrm{W}$ Tir nehmen zunächst an, daß für die Elektronen eines Halbleiters nur zwei Zustände (Energieniveaus) 1 und 2 der statistischen Gewichte $N_{1}$ und $N_{2}$ und der Energien $E_{1}$ und $E_{2}$ möglich sind, etwa das Grundband und das Leitungsband ohne Berücksichtigung von Störstellen usw. Von vornherein wird angenommen, daß $E_{2}$ mindestens um einige $k T$ größer sei als $E_{1}$. Wir fragen nach den Häufigkeiten der Übergänge $1 \rightarrow 2$ bzw. $2 \rightarrow 1$, beide sind im thermodynamischen Gleichgewicht einander gleich. Die Übergänge werden hervorgerufen durch die Wechselwirkung mit den Schallquanten der Gitterschwingungen. Ein Übergang $\mathbf{1} \rightarrow \mathbf{2}$ ist nur möglich, wenn ein Schallquant die Energie $\Delta E=E_{2}-E_{1}$ abgibt, also aus einem Zustand $k$ der Energie $E_{k}$ übergeht in einen Zustand $k^{\prime}$ der Energie $E_{k^{\prime}}$, wobei $E_{k}-E_{k^{\prime}}=\Delta E$ sein muß.

Nach allgemeinen quantenstatistischen Grundsätzen ist die Zahl der Übergänge von Elektronen von 1 nach 2 bei gleichzeitigem Übergang ebenso vieler Schallquanten von $k$ nach $k^{\prime}$ gegeben durch

$$
w_{1 k}^{2 k^{\prime}} n_{1} n_{k} f_{2}^{(\mathrm{e})}\left(n_{2}\right) f_{k^{\prime}}^{(\mathrm{s})}\left(n_{k^{\prime}}\right)^{1}
$$

Dabei ist $w_{1 k}^{2 k^{\prime}}$ die Elementarwahrscheinlichkeit für den Prozeß $1 \rightarrow 2$ mit gleichzeitigem $k \rightarrow k^{\prime}, n_{k}$ bzw. $n_{k^{\prime}}$ die Zahl der Schallquanten im Zustand $k$ bzw. $k^{\prime}, n_{1}$ bzw. $n_{2}$ die Zahl der Elektronen im Zustand 1 bzw. 2. $f^{(\mathrm{e})}$ und $f^{(\mathrm{s})}$ kennzeichnen die Abhängigkeit der Übergangswahrscheinlichkeit von der Besetzung des Endzustandes. Es ist $f_{2}{ }^{(\mathrm{e})}=N_{2}-n_{2}$ (Fermi-Statistik für die Elektronen) und $f_{k^{\prime}}^{(\mathrm{s})}=g_{k^{\prime}}$

1 E. Madelung, Die mathematischen Hilfsmittel des Physikers, 4. Auflage, Göttingen 1950, S. 494, umgeschrieben auf unsere Bezeichnungen. $+n_{k^{\prime}}$ (Bose-Statistik für die Schallquanten). $g_{k^{\prime}}$ ist die Zahl der Phasenraumzellen mit $E_{k^{\prime}}$. Für die uns interessierenden Übergänge $1 \rightarrow 2$ ist es gleichgültig, welcher Übergang $k \rightarrow k^{\prime}$ für die Erfüllung des Energiesatzes sorgt, wir summieren daher (1) über alle $k$ und $k^{\prime}$, für die die Bedingung

$$
E_{k}-E_{k^{\prime}}=E_{2}-E_{1}=\Delta E
$$

gilt, und erhalten

$$
n_{1}\left(N_{2}-n_{2}\right) \sum_{k k^{\prime}} w_{1 k}^{2 k^{\prime}} n_{k}\left(g_{k^{\prime}}+n_{k^{\prime}}\right) .
$$

Schreibt man für die Summe zur Abkürzung $w_{12}$, so ist

$$
w_{12}=\sum_{k k^{\prime}} w_{1 k}^{2 k^{\prime}} \frac{g_{k}}{e^{E_{k} / k T}-1} \frac{g_{k^{\prime}} e^{E_{k^{\prime}} / k T}}{e^{E_{k^{\prime}} / k T}-1},
$$

da für die Schallquanten Bose-Statistik gilt, also

$$
n_{i}=\frac{g_{i}}{e^{E_{i} / k T}-1}\left(i=k, k^{\prime}\right) .
$$

Aus (2) folgt dann

$$
w_{12}=e^{-\Delta E / k T} \sum_{k k^{\prime}} w_{1 k}^{2 k^{\prime}} \frac{g_{k} g_{k^{\prime}}}{\left(1-e^{-E_{k} / k T}\right)\left(e^{E_{k^{\prime}} / k T}-1\right)}
$$

Da $\Delta E$ sehr groß gegen $k T$ sein soll, ist sicher $E_{k} \gg k T$; andererseits liefern die Glieder der Summe, für die $E_{k^{\prime}}$ klein ist, den größten Beitrag. Wenn wir daher schreiben

$$
w_{12}=w e^{-\Delta E / k T},
$$

so wird $w$ relativ langsam mit der Temperatur veränderlich sein, verglichen mit der stark temperaturabhängigen Exponentialfunktion. Im übrigen hängt $w$ zwar von den mechanischen, insbesondere den elastischen Eigenschaften des betrachteten Halb- 
leiters und den quantentheoretischen Elementarwahrscheinlichkeiten, nicht aber von den charakteristisch elektrischen Größen wie Bandabstand, Störstellenkonzentration usw. ab. Wir betrachten $w$ für das Weitere als eine Konstante.

Die vorstehenden Überlegungen gelten analog für den Prozeß $2 \rightarrow 1$ mit Vertauschung des Indizes 1 und 2 bzw. $k$ und $k^{\prime}$. An die Stelle der Gl. (4) tritt also

$$
w_{21}=\sum_{k k^{\prime}} w_{2 k^{\prime}}^{1 k} \frac{g_{k^{\prime}}}{e^{E_{k^{\prime}} / k T}-1} \frac{g_{k} e^{E_{k} / k T}}{e^{E_{k} / k T}-1} .
$$

Da aber ganz allgemein $w_{1 k}^{2 k^{\prime}}=w_{2 k^{\prime}}^{1 k}$, so folgt aus (8) sofort

$$
w_{21}=w \text {. }
$$

Aus der Gleichsetzung der Anzahlen von Übergängen $1 \rightarrow 2$ und $2 \rightarrow 1$ im Gleichgewicht

$$
w_{12} n_{1}\left(N_{2}-n_{2}\right)=w_{21} n_{2}\left(N_{1}-n_{1}\right)
$$

folgt durch Einsetzen von (7) und (9) unmittelbar die Fermi-Verteilung

$$
n_{i}=\frac{N_{i}}{1+e^{\frac{E_{i}-5}{k T}}}(i=1,2)^{2},
$$

wie man sich am einfachsten durch Einsetzen überzeugt, für jedes $\zeta$. $\zeta$ bestimmt sich durch die Bedingung

$$
n_{1}+n_{2}=\text { const. }
$$

Fügt man auf der linken Seite der Gl. (10) eine Anzahl von $J$ Elektronen hinzu, die pro Zeiteinheit in das höhere Energieniveau 2 gehoben werden, so hat man den Fall des inneren Photoeffekts:

$$
J+w_{12} n_{1}\left(N_{2}-n_{2}\right)=w_{21} n_{2}\left(N_{1}-n_{1}\right) .
$$

Bezeichnet man mit $n_{10}$ und $n_{20}$ die Elektronenzahlen im ungestörten Zustand, so ist

$$
\text { und } \quad n_{2}=n_{20}+\varepsilon_{2} \text {, }
$$

wenn $\varepsilon_{1}$ und $\varepsilon_{2}$ die Zahlen der durch den lichtelektrischen Effekt zusätzlich gebildeten Elektronen bezeichnen. Solange man sich auf zwei Niveaus beschränkt, muß aber nach wie vor die Beziehung (12) gelten, d. h. es muß $-\varepsilon_{1}=\varepsilon_{2}=\varepsilon$ sein. Berücksichtigt man dies und setzt man die $w_{i k}$ aus (7) und (9), die $n_{i 0}$ aus (11) ein, so folgt

$w \varepsilon^{2}\left(1-\frac{1}{A}\right)+w \varepsilon\left(\frac{N_{1}}{A} \frac{A+X}{1+X}+N_{2} \frac{1+X}{A+X}\right)=J$

${ }^{2}$ R. Peierls, Ergebn. exakt. Naturwiss. 11, 264 [1932]. mit den Abkürzungen

und

$$
e^{E_{2} / k T} \equiv A
$$$$
e^{\zeta / k T} \equiv X \text {. }
$$

Dabei sind alle Energieabstände von $E_{1}$ aus gezählt.

Gl. (15) stellt den einfachsten denkbaren Fall eines inneren Photoeffektes dar, bei dem nur zwei Energieniveaus wirksam werden. Damit die Änderung der Besetzungszahlen als Leitfähigkeitsänderung merklich wird, muß mindestens eines der beiden beteiligten Niveaus das Grundband oder das Leitungsband sein.

Die gemachten Ansätze lassen sich offenbar auch auf kompliziertere Modelle übertragen, die mehr als zwei Niveaus, z. B. noch Haftstellen, Aktivatorterme usw. betrachten. Man hat dann die Annahme aufzugeben, daß jeder Prozeß in beiden Richtungen gleich häufig erfolgt, denn der Photoeffekt hebt das thermodynamische Gleichgewicht auf. In Gl. (13) gilt diese Gleichsetzung noch, weil überhaupt nur das Wechselspiel zwischen zwei Termen betrachtet wird. Aber auch bei mehreren Termen stellt sich nach Ablauf des Anklingens eine stationäre Besetzung der einzelnen Terme ein, und die Gesamtzahl der ein bestimmtes Niveau erreichenden Elektronen muß dann wieder gleich der Zahl der Elektronen sein, die dieses Niveau verlassen. So ergibt sich eine „,Elektronenbilanz“, die z. B. im Falle von drei Niveaus so aussieht:

$$
\begin{gathered}
w_{21} n_{2}\left(N_{1}-n_{1}\right)+w_{31} n_{3}\left(N_{1}-n_{1}\right) \\
=w_{12} n_{1}\left(N_{2}-n_{2}\right)+w_{13} n_{1}\left(N_{3}-n_{3}\right)+J \\
J+w_{12} n_{1}\left(N_{2}-n_{2}\right)+w_{32} n_{3}\left(N_{2}-n_{2}\right) \\
\quad=w_{21} n_{2}\left(N_{1}-n_{1}\right)+w_{23} n_{2}\left(N_{3}-n_{3}\right) \\
w_{13} n_{1}\left(N_{3}-n_{3}\right)+w_{23} n_{2}\left(N_{3}-n_{3}\right) \\
=w_{31} n_{3}\left(N_{1}-n_{1}\right)+w_{32} n_{3}\left(N_{2}-n_{2}\right) .
\end{gathered}
$$

Die Gl. $(16 \mathrm{a}-\mathrm{c})$ stellen in dieser Reihenfolge die Bilanzen für die Niveaus 1, 2, 3 dar, links stehen Zugänge, rechts Abgänge an Elektronen. Es ist angenommen, daß der Photoeffekt $J$ Elektronen pro Zeiteinheit vom Niveau 1 in das Niveau 2 hebt. $J$ erscheint also beim Niveau 1 als Abgang, beim Niveau 2 als Zugang. (16c) entsteht offenbar durch Addition aus (16a) und (16b). Die dritte unabhängige Gleichung für die Berechnung der $n_{i}$ liefert

$$
n_{1}+n_{2}+n_{3}=\text { const., }
$$

d. h. die Zahl der zur Verfügung stehenden Elektronen ist konstant. 


\section{Eigensahaften der Prozesse mit zwei Niveaus}

Vorläufig verzichten wir noch darauf, den. beteiligten Niveaus besondere Bedeutungen als Grundband, Leitungsband, Störterm u. dgl. beizulegen.

Zunäehst ist ohne weiteres ersichtlich, daß in Gl. (15) für nicht zu große $J$ das in $\varepsilon$ quadratische Glied zu vernachlässigen ist. Man erhält dann eine Gleichung vom Typ

$$
\alpha \varepsilon=J,
$$

wobei $\alpha=1 / \tau$ die Zeitkonstante der durch das Licht hervorgerufenen Störungen wird. Offenbar wird nach Aufhören der Bestrahlung die Elektronenverteilung nach

$$
\frac{\mathrm{d} \varepsilon}{\mathrm{d} t}=-\alpha \varepsilon
$$

in den Ausgangszustand zurückkehren, wenn die Bestrahlung nicht so stark war, daß das quadratische Glied ins Gewicht fällt.

Aus Gl. (11) ist abzulesen, daß die Differenz $\varepsilon$ der Leitungsträgerzahlen für ein gegebenes $J$ um so gröBer ist, je kleiner $\alpha, d$. h. je größer $\tau$ wird. Es ist also gleichgültig, ob man die Wirksamkeit des Photoeffekts oder die Zeitkonstante des Prozesses untersucht.

Vor allem wird nun die Frage interessieren, unter welchen Bedingungen $\alpha$ einen möglichst kleinen Wert annimmt. Die zweite Klammer in Gl. (15) hat ihr Minimum für

$$
X=\sqrt{A} \frac{\sqrt{N_{1} A}-\sqrt{N_{2}}}{\sqrt{N_{2} A}-\sqrt{N_{1}}} .
$$

In einem Halbleiter hat man es im allgemeinen mit energetischen Abständen zu tun, die groß gegen $k T$ sind. $A$ wird also eine sehr große Zahl sein, z. B. für einen Niveauabstand von $1 \mathrm{eV}$ bei Zimmertemperatur etwa $10^{17}$. Wenn also $N_{1}$ und $N_{2}$ nicht um viele Größenordnungen verschieden sind, verschwinden die Subtrahenden im Zähler und Nenner von (19) und man erhält

$$
X=\sqrt{A} \sqrt{\frac{N_{1}}{N_{2}}}
$$

oder durch Logarithmieren

$$
\zeta=\frac{E_{2}}{2}+\frac{k T}{2} \ln \left(N_{1} / N_{2}\right) .
$$

Solange das Verhältnis von $N_{1}$ zu $N_{2}$ nicht so groß ist, daß sein natürlicher Logarithmus $\gg 1$ ist, hat die Störung die höchste Lebensdauer, wenn die Fermi-Kante in der Mitte zwischen den beiden Niveaus liegt.
Das durch die Bedingung (19) gegebene Maximum der Lebensdauer ist sehr scharf. Für einen einfachen Fall ist in Abb. 1 die Abhängigkeit der Größe $w \tau$, die der photoelektrischen Wirksamkeit bzw. der Lebensdauer der Störung proportional ist, von der Grenzenergie $\zeta$ aufgetragen. Als Niveaus sind das Grundband und das Leitungsband selbst angenommen mit einem willkürlich gewählten Bandabstand von $1,84 \mathrm{eV}$.

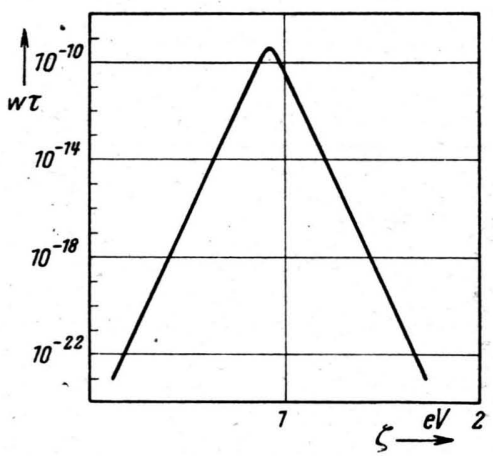

Abb. 1. Abhängigkeit der photoelektrischen Wirksamkeit von der Lage der Fermi-Kante bei zwei beteiligten Niveaus.

Das einfache Modell, das der Abb. 1 zugrunde liegt, sei noch etwas näher betrachtet. Die Leitungsvorgänge sind bei diesem Modell gegeben durch die Besetzung eines Leitungsbandes mit Elektronen und eines Grundbandes mit Löchern, im wesentlichen. liegt also ein Eigenhalbleiter vor. Es sei jedoch zugelassen, daß der Halbleiter beim absoluten Nullpunkt eine Leitfähigkeit besitzt, d. h. daß entweder ,thermisch inaktive" Störstellen im Sinne von Gisolf ${ }^{3}$ vorhanden sind, oder daß das thermodynamische Gleichgewicht in anderer Weise gestört wird. Man kann dabei an Randschichten, Korngrenzen u. ä. denken. Dadurch können Verhältnisse geschaffen sein, die örtlich einen Quasi-Eigenhalbleiter erzeugen, auch wenn das kompakte Material diese Eigenschaft nicht besitzt.

Die Niveaus 1 und 2 aus Gl. (10) sind jetzt das Grund- und das Leitungsband mit dem statistischen Gewicht $N_{0}=\left(\frac{m^{*} k T}{h \hbar}\right)^{3 / 2}\left(\right.$ vgl. $\left.{ }^{3}\right)$, für die Scheinmasse $m^{*}$ sei in beiden Bändern die wirkliche Elektronenmasse gesetzt. Zahlenmäßig ergibt sich dann bei Zimmertemperatur $N_{0}=1,215 \cdot 10^{25} \cdot \mathrm{m}^{-3}$. Ein Maß für die Leitfähigkeit ist $n+p$, die Summe aus

\footnotetext{
3 J. H. Gisolf, Ann. Physik 1, 1 [1947].
} 
der Konzentration der freien Elektronen $n=n_{2}$ und der Zahl der Defektelektronen im Grundband $p=N_{0}-n_{1}$. Eine eventuelle Verschiedenheit der Beweglichkeiten ist also nicht berücksichtigt. Am allgemeinen Charakter der Funktionen ändert sich dadurch nichts Wesentliches.

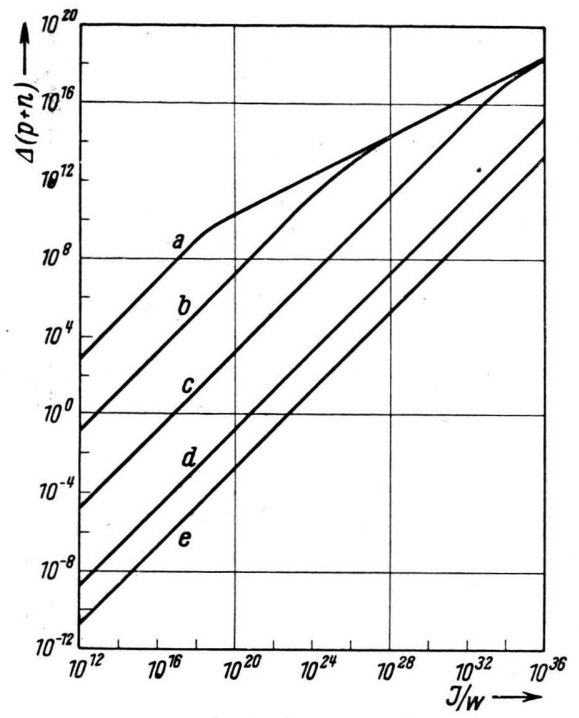

Abb. 2. Abhängigkeit des Photostroms von der Bestrahlungsstärke im Eigenhalbleiter für verschiedene Lagen der Fermi-Kante. Werte für $\zeta$ in $\mathrm{eV}:$ a) 0,92; b) 0,69 oder 1,15 ; c) 0,46 oder 1,38 ; d) 0,23 oder 1,61 ; e) 0,115 oder 1,725 .

Mit diesen Annahmen sind die Kurven der Abb. 2 berechnet. Aufgetragen ist die Differenz von $(p+n)$ mit und ohne Bestrahlung gegen die der Bestrahlungsstärke proportionale Größe $J / w$. Der Photostrom ist für geringe Bestrahlung der Bestrahlungsstärke proportional, für sehr starke Bestrahlung proportional ihrer Wurzel. Der Übergang von der linearen zur Wurzelabhängigkeit findet statt, wenn der Photostrom größer wird als der Dunkelstrom. Für hohe Bestrahlungsstärken laufen die Kurven in einer Graden zusammen, für kleine Bestrahlungsstärken ist die photoelektrische Wirksamkeit um so größer, je genauer die Fermi-Kante in der Mitte zwischen den Bändern liegt.

\section{Modelle mit drei beteiligten Niveaus}

Erweitert man das bisher betrachtete Bild durch ein diskretes Störniveau des statistischen Gewichtes $N$, so entsteht an Stelle der bisherigen Gl. (13) ein Gleichungssystem, das die Übergänge zwischen dem Grundband, dem Leitungsband und dem an- genommenen Störniveau enthält. Grundsätzlich kann für jeden dieser Übergänge ein anderes $w$ maßgebend sein. Als charakteristische Fälle werden folgende behandelt: a) alle drei $w$ sind gleich; b) der Übergang vom Störniveau zum Grundband ist verboten, d. h. für ihn ist $w$ gleich Null. Der unter b) genannte Fall entspricht einem typischen Haftterm, für den die Rekombination mit den Löchern des Grundbandes verboten ist. Betrachten wir mit Schö ${ }^{4}$ die Anlagerung von Löchern an derartige Haftterme als typischen ,,strahlungslosen Übergang", so entspricht also der Fall b) einem Phosphor, bei dem die strahlungslosen Übergänge unterdrückt sind.

In dem unter a) genannten Fall ergeben sich aus Gl. (16) nach elementarer Umrechnung die „Bilanzgleichungen":

$$
\begin{aligned}
& x \frac{N_{0}}{X}+y N_{0}\left(\frac{1}{B}+\frac{1}{X}\right)+z\left(N_{0} \frac{X}{A}+N \frac{X}{B+X}\right) \\
& +z(y+x)=J / w, \\
& x N \frac{B}{B+X}-y N_{0} \frac{(B X+A)(B+X)}{A B X} \\
& -z N \frac{X}{B+X}-y(x+z)=0, \\
& J / w=x\left(\frac{N_{0}}{X}+N \frac{B}{B+X}\right)-y N_{0} \frac{B+X}{A} \\
& +z N_{0} \frac{X}{A}+x(z-y), \\
& x+y=z \text {. }
\end{aligned}
$$

Hierbei ist für Grund- und Leitungsband wieder das statistische Gewicht $N_{0}$ eingesetzt, für das Störniveau ein statistisches Gewicht $N$ entsprechend der Konzentration der Störstellen. $x$ ist die Abweichung der Elektronenzahl $n$ im Leitungsband von dem Wert $n_{0}$ ohne Belichtung; entsprechend ist $y$ die Abweichung der Elektronenzahl $v$ im Störniveau von dem Wert $v_{0}$ ohne Belichtung und $z$ die Abweichung der Defektelektronenzahl $p$ im Grundband von dem Wert $p_{0}$ ohne Belichtung. Zur Abkürzung ist gesetzt $B \equiv e^{E_{\mathrm{S}} / k T}$, wenn $E_{\mathrm{S}}$ den Energieabstand zwischen Grundband und Störterm bezeichnet. Schließlich sind für $n_{0}, v_{0}$ und $p_{0}$ die Besetzungszahlen gemäß (11) eingeführt.

Das Gleichungssystem (22), das nächst dem Eigenhalbleiter den einfachsten denkbaren Fall darstellt, führt für $x, y, z$ als Funktion von $J$ bereits auf eine Gleichung 4. Grades. Man kommt aber mit

\footnotetext{
${ }^{4}$ M. Schön, Z. Naturforschg. 6a, 215 [1951].
} 
quadratischen Gleichungen aus, wenn man z. B. $y$ als Parameter vorgibt und dann $J, x$ und $z$ als Funktion von $y$ berechnet. Auf diese Weise sind die Kurven für Abb. 3 ermittelt, die in einem Beispiel mit willkürlichen Zahlenannahmen die wesentlichen $\mathrm{Zu}$ sammenhänge zeigt. Die Leitfähigkeitsänderung ist etwa proportional der Summe $x+z$; genauer wäre es, $b_{n} x+b_{p} z$, d. h. die Summe der Produkte aus Trägerzahl und Beweglichkeit, einzusetzen. Hier ist $x+z$ als Funktion von $J$ für verschiedene Lagen der Fermi-Kante aufgetragen.

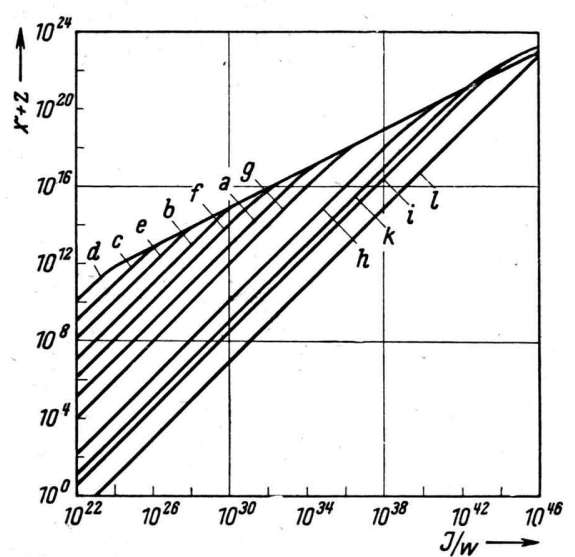

Abb. 3. Abhängigkeit des Photostroms von der Bestrahlungsstärke im Halbleiter mit einem Haftniveau für verschiedene Lagen der Fermi-Kante. Strahlungslose Übergänge erlaubt. Werte für $\zeta$ in $\mathrm{eV}$ : a) 0,46 ; b) 0,575 ; c) 0,69 ; d) 0,805 ; e) 0,92 ; f) 1,035 ; g) 1,15 ;

h) 1,265 oder 1,495 ; i) 1,38 ; k) 1,61 ; 1) 1,725 .

Wie in Abb. 2 ist der Zusammenhang zwischen Photostrom und Bestrahlungsstärke für kleine Bestrahlungsstärken linear, für sehr große Bestrahlungsstärken besteht ein Wurzelgesetz. Der Übergang zwischen diesen beiden Gebieten findet aber jetzt nicht mehr dann statt, wenn der Hellstrom gleich dem Dunkelstrom geworden ist, vielmehr reicht das lineare Gebiet noch zu höheren Bestrahlungsstärken. Auch ergeben sich die größten Photoströme nicht mehr für den Fall, daß die FermiKante gerade in der Mitte zwischen den Bändern liegt. Wenn man die Stromänderung für kleine Bestrahlungsstärken betrachtet, so läßt sich die günstigste Lage berechnen; wegen der Umständlichkeit der Rechnung sei nur das Ergebnis mitgeteilt: die stärkste Stromänderung ergibt sich für

$$
X=\sqrt{A} \frac{1}{\sqrt{1+\frac{N A}{N_{0} B}}},
$$

d. i. logarithmiert für

$$
\zeta=\frac{E_{2}}{2}-\frac{k T}{2} \ln \left(1+\frac{N A}{N_{0} B}\right) .
$$

Wenn man allerdings nicht die Leitfähigkeitsänderung, sondern das Verhältnis der Stromänderung zum Dunkelstrom betrachtet, so geht mit der Verschiebung der Fermi-Kante von der Mitte zwischen den Bändern in die durch (23a) gekennzeichnete günstigste Lage auch eine Erhöhung der Dunkelleitfähigkeit im gleichen Maß vor sich, und der Quotient beider bleibt etwa konstant. Das würde dem experimentellen Befund entsprechen, daß man bei Photowiderständen eines bestimmten Typs (etwa $\mathrm{CdS}$ oder $\mathrm{PbS}$ ) hoch- und niederohmige Exemplare findet, bei denen das Verhältnis von Photostrom zu Dunkelstrom etwa gleich günstig ist.

Im Fall b) des verbotenen Übergangs zwischen Haftniveau und Bandrand lauten die „Bilanzgleichungen"

$$
\begin{gathered}
x \frac{N_{0}}{X}+z N_{0} \frac{X}{A}+x z=\frac{J}{w}, \\
x N \frac{B}{B+X}-y N_{0} \frac{B+X}{A}-x y=0 .
\end{gathered}
$$

$(24 \mathrm{c})$ und $(24 \mathrm{~d})$ sind mit $(22 \mathrm{c})$ und $(22 \mathrm{~d})$ identisch, in $(24 a)$ und $(24 b)$ sind die Glieder weggefallen, die von dem Übergang zwischen Grundband und Haftterm herrühren. Es gilt wie im Falle a) lineare Abhängigkeit bei kleinen, Wurzelabhängigkeit bei sehr großen Bestrahlungsstärken, dazwischen schiebt sich jetzt aber bei gewissen Lagen der Fermi-Kante

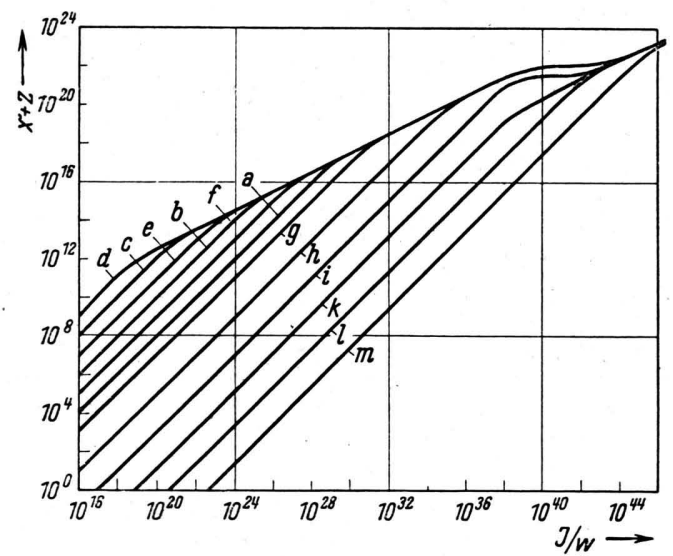

Abb. 4. Abhängigkeit des Photostroms von der Bestrahlungsstärke im Halbleiter mit einem Haftniveau für verschiedene Lagen der Fermi-Kante. Strahlungslose Übergänge verboten. Werte für $\zeta$ in $\mathrm{eV}:$ a) 0,46 ; b) 0,575 ; c) 0,69 ; d) 0,805 ; e) 0,92 ; f) 1,035 ; g.) 1,15 ; h) 1,265 ; i) 1,38 ; k) 1,495 ; 1) 1,61 ; m) 1,725 . 
ein Übergangsgebiet, wie die Betrachtung der Abb. 4 lehrt, die mit den gleichen angenommenen Zahlenwerten berechnet ist wie Abb. 3. Einzelne Kurven laufen stückweise etwa parallel zur Abszissenachse, d. h. daß eine weitere Erhöhung der Bestrahlungsstärke keine weitere Erhöhung des Photostroms bewirkt. Dieser Fall ist experimentell nicht bekannt. Er bedeutet anschaulich, daß der Photoeffekt zwischen Grundband und Haftniveau aufgehört hat, weil das Haftniveau vollkommen besetzt ist. Eine weitere Steigerung des Photostromes ist dann erst wieder möglich, wenn bei extrem starken Bestrahlungen der Photoeffekt zwischen Grundband und Leitungsband analog Abschnitt 1 einsetzt. Daraus geht hervor, daß das Modell mit einem diskreten Haftniveau zu einfach ist, oder daß experimentell so große Bestrahlungsstärken nicht erreichbar sind.

\section{Behandlung komplizierter Modelle und der An - und Abklingvorgänge}

In den vorhergehenden Abschnitten sind die einfachsten denkbaren Modelle eines Photohalbleiters ausführlicher besprochen. Eine Erweiterung auf kompliziertere Modelle ist möglich, indem man in den Ansatz (22) weitere Terme einführt. Allerdings werden die Gleichungen dann sehr kompliziert. Denkt man außer diskreten Störtermen noch an kontinuierlich verteilte Niveaus (,,Haftstellenspektrum"), so treten an die Stelle bestimmter statistischer Gewichte $N$ Termdichten $D(E)$ auf der Energieskala, und eine analoge Behandlung ist ohne weiteres möglich; ein Beispiel für eine exponentielle Verteilung $D(E)$ zur Deutung des gebrochenen Exponenten in der Intensitäts-Photostrom-Abhängigkeit des CdS wird an anderer Stelle besprochen ${ }^{5}$.
Auch die Abhängigkeit des Photostroms von der Zeit beim Ein- und Abschalten der Bestrahlung ist mit den Ansätzen (22) zu behandeln. Die ,Elektronenbilanz" entsteht im stationären Zustand für jeden Term dadurch, daß die Zahl der den Term erreichenden Elektronen in der Zeiteinheit gleichgesetzt wird der Zahl der Elektronen, die den betreffenden Term verlassen. Bevor dieser stationäre Zustand erreicht ist, bedeutet der Überschuß der ankommenden über die weggehenden Elektronen die zeitliche Vermehrung der Elektronenzahl auf dem betreffenden Term. Man erhält so ein System von Differentialgleichungen 1. Ordnung, die alle untereinander abhängig sind. Wegen der vorkommenden Produkte der Besetzungszahlen verschiedener Terme sind die Differentialgleichungen nicht linear und daher im allgemeinen nur durch Näherungsmethoden lösbar. Für geringe Bestrahlungsstärken, bei denen diese Produkte vernachlässigbar sind, bekommt man $\beta-1$ lineare Differentialgleichungen, falls $\beta$ Terme betrachtet werden, dazu eine Gleichung nach Art von (22d), die die Konstanz der Zahl der am Prozeß beteiligten Elektronen bedeutet. Die Lösungen sind dann näherungsweise $\beta-1$ sich überlagernde Exponentialfunktionen mit im allgemeinen sehr verschiedenen Zeitkonstanten. Es besteht also kein Grund, die kürzesten dieser Abklingzeiten einem Primärstrom, alle übrigen Sekundärströmen zuzuordnen. Vielmehr entsprechen die verschiedenen Abklingzeiten näherungsweise den Einstellzeiten für die verschiedenen beteiligten Prozesse, die aber alle lichtelektrischer Natur sind.

5 Vortrag des Verf. auf der Festkörpertagung in Dresden, 9.-11. 5. 1952, Referat im Druck. 